Pirineos, 164: 135 a 164, JACA; 2009. ISSN 0373-2568

\title{
MÉTODO ESPECÍFICO PARA LA EVALUACIÓN MEDIOAMBIENTAL DE LOS LAGOS DE ORIGEN GLACIAR PIRENAICOS Y SU APLICACIÓN AL LAGO DE SABOCOS
}

\author{
T. Arruebo ${ }^{1}$, A. Pardo ${ }^{2}$, C. Rodríguez ${ }^{3}$, F. J. Lanaja ${ }^{4}$ \& J. del Valle ${ }^{1}$ \\ 1 Departamento de Geografía y Ordenación del Territorio, Facultad de Filosofía y Letras, \\ Universidad de Zaragoza. C/Pedro Cerbuna, 12, E-50009, Zaragoza, España. \\ 2 Departamento de Buceo Científico y Medio Ambiente, Federación Aragonesa de Actividades \\ Subacuáticas (FARAS), Padre Marcellán, 15 (Edificio Kasan) 50015, Zaragoza, España. \\ 3 Colegio Oficial de Físicos. C/Monte Esquinza, 28, E-28010, Madrid, España. \\ 4 Laboratorio de Calidad de Aguas, Escuela Universitaria de Ingeniería Técnica Industrial \\ de Zaragoza. C/María Luna, 3, E-50015, Zaragoza, España. \\ tarruebo@gmail.com
}

\begin{abstract}
This study proposes a new method to asses the environmental state of the Pyrenean glacial lakes, based on the Water Framework Directive, the concept of ecological state and inspired by widely tested and used methodologies. Starting from a good ecological state as a reference term, a series of matrices are used to identify and characterize all anthropic impacts and pressures. Information regarding the most significant ones is then compared with the criteria of a panel of experts and finally the results are summarised in ICPA matrices of Impacts, Consequences, Proposals of Corrective Measures, and Applicability. This method has been tested in the glacial lake of Sabocos, finding that its ecological quality is lower than expected, based on the identification of some severe impacts. In order to subdue them, it has been proposed a plan of correcting measures and valued its applicability.
\end{abstract}

Keywords: Environmental assessment, Water Framework Directive, significant impacts, ICPA matrix, glacial lakes, Pyrenees.

RESUMEN.- En este trabajo se propone un nuevo método para la evaluación medioambiental de los lagos pirenaicos de origen glaciar a partir de la Directiva Marco del Agua, fundamentado en el concepto de estado ecológico e inspirado en metodologías ampliamente contrastadas. Partiendo de un estado ecológico de referencia se identifican y caracterizan todas las presiones e impactos antropogénicos mediante el empleo de una serie de matrices adaptadas. Los impactos más significativos son contrastados por un 


\section{T. ARRUEBO, A. PARDO, C. RODRÍGUEZ, F. J. LANAJA \& J. DEL VALLE}

panel de expertos. Finalmente, los resultados se expresan mediante matrices ICPA de Impactos, Consecuencias, Propuestas de medidas correctoras y Aplicabilidad. Este método de evaluación medioambiental se ha aplicado al lago de Sabocos. Los resultados obtenidos muestran una calidad ecológica inferior a la esperada y que algunos de los impactos identificados son severos. Con el objetivo de mitigar tales afecciones, se han propuesto una serie de medidas correctoras y evaluado su aplicabilidad.

Palabras clave: Evaluación medioambiental, Directiva Marco del Agua, impactos significativos, matriz ICPA, lagos de origen glaciar, Pirineos.

\section{Introducción y objetivos}

El 23 de octubre de 2000 se publicó la Directiva 2000/60/CE, del Parlamento Europeo y del Consejo, por la que se establece un marco comunitario de actuación en el ámbito de la política de aguas, denominada Directiva Marco del Agua (DMA), que tiene como objetivo que todas las aguas superficiales de la Comunidad Europea alcancen un buen estado químico y ecológico antes del 2015 (cf., PREÁMBULO, PUNTOS 25, 26). Con esta finalidad, una de las medidas que establece es la necesidad de realizar un estudio de las repercusiones de la actividad humana en el estado de las aguas (cf., ARTÍCULO 5).

Para ello, se ha de recoger información del total de presiones significativas a las que se vea expuesta la masa de agua ( $c f$. ., ANEXO II, 1.4), y evaluar la susceptibilidad del estado ecológico respecto al total de presiones identificadas ( $c f$. ., ANEXO II, 1.5). Posteriormente, debe implantarse un plan de medidas correctoras en función de las repercusiones analizadas (cf., ARTíCULO 11). Todo ello hace necesario elaborar metodologías específicas para cada tipo de masa de agua que faciliten su correcta evaluación.

El objetivo de este artículo es proponer un método sencillo y específico para la evaluación medioambiental de los lagos pirenaicos fundamentado en la DMA. Posteriormente se aplica esta metodología a un caso concreto, el lago de Sabocos. Los resultados permiten establecer las pautas necesarias para la correcta gestión de este entorno.

\section{Método propuesto}

2.1. Descripción del área de estudio: delimitación y caracterización de la masa de agua (cf., DMA, ANEXO II, APARTADO 1.1.)

El área de estudio quedará delimitada por la cota máxima de la lámina de agua (Fig. 1). Se aportará información geográfica básica: Red hidrográfica y 
Región Biogeográfica a la que pertenece, coordenadas UTM, breve explicación de sus accesos y plano de localización.

Para la caracterización de la masa de agua se emplean los criterios expuestos por la Agencia Catalana del Agua (ACA, 2006), que clasifican los lagos en función de su origen, geología, altitud y alcalinidad de sus aguas.

\subsection{Evaluación de la situación actual del ecosistema: calidad ecológica}

Dos son los problemas que presenta la obtención del estado ecológico tal y como se define en la DMA. Por una parte, en la actualidad no existen índices específicos para evaluar todos los indicadores que la DMA propone para la determinación del estado ecológico. Por otra, algunos para los que existe bibliografía requieren grandes recursos materiales y humanos. Por tanto, para abordar este problema de una manera efectiva se empleará el concepto de calidad ecológica (cf., LANAJA et al., 2005), que es una buena aproximación al de estado ecológico (ver tabla 1).

Tabla 1. Calidad Ecológica, variables valoradas y métricas empleadas para ello. Table 1. Ecological quality, variables used and indices used.

\begin{tabular}{|c|c|c|c|}
\hline Elementos & Variables & Parámetros & Métricas \\
\hline \multirow[t]{2}{*}{ Biológicos: } & $\begin{array}{c}\text { Invertebrados } \\
\text { bentónicos }\end{array}$ & $\begin{array}{l}\text { Macroinvertebrados } \\
\text { bentónicos }\end{array}$ & $\begin{array}{l}\text { Índice IBMWP (ALBA TERCE- } \\
\text { DOR y SÁNCHEZ ORTEGA, } \\
\text { 1988). } \\
\text { Índice InMacro (ACA, 2006). }\end{array}$ \\
\hline & Fauna íctica & Peces & Presencia/ausencia (ACA, 2006). \\
\hline Fisicoquímicos & $\begin{array}{c}\text { Características } \\
\text { F-Q }\end{array}$ & 42 parámetros & $\begin{array}{l}\text { Calidad Teórica (CHE,1990). } \\
\text { Calidad del Agua de Adour- } \\
\text { Garonne (AGENCE DE L’EAU } \\
\text { ADOUR-GARONNE, 2005). } \\
\text { Límites de Calidad propuestos } \\
\text { por la Agencia Catalana del Agua } \\
\text { (ACA, 2006). } \\
\text { Índice IPO (LECLERCQ, 2001). }\end{array}$ \\
\hline
\end{tabular}

La evaluación de las variables propuestas por la DMA para la determinación del estado ecológico se realiza a partir de distintas métricas. En la tabla se sintetizan las variables consideradas en este estudio y las métricas empleadas para ello. 
Para la determinación de la calidad ecológica se emplean algunos de los indicadores tradicionalmente utilizados para la evaluación del estado de cualquier tipo de masa de agua superficial. Son sencillos e implican un bajo coste, y la principal ventaja de su uso es dar una visión adecuada del estado del sistema con pocos recursos (RODRÍGUEZ et al., 2009). No obstante, la consideración de un mayor número de los indicadores específicos para lagos propuestos por la DMA daría una visión más precisa y próxima al estado ecológico del ecosistema.

- Métricas empleadas para la determinación de la calidad biológica ( $c f$., RODRÍGUEZ et al., 2009):

- Fauna bentónica de invertebrados:

- En los puntos de aguas lóticas se aplica el índice IBMWP (ALBA TERCEDOR y SÁNCHEZ ORTEGA, 1988), considerando las ecorregiones de la cuenca del Ebro (cf., PRAT y MUNNE, 1999).

- Y en el conjunto del lago, unificando el total de puntos en una única muestra, se aplica el índice InMacro (ACA, 2006).

- Fauna íctica: presencia/ausencia de peces (ACA, 2006).

- Métricas empleadas para la determinación de la calidad fisicoquímica:

- Calidad Teórica de la Confederación Hidrográfica del Ebro (CHE, 1990).

- Calidad del Agua de Adour-Garonne (ADOUR-GARONNE, 2005).

- Límites de Calidad propuestos por la Agencia Catalana del Agua (ACA, 2006).

- Índice de Polución Orgánica (IPO). (LECLERCQ, 2001).

\section{- Calidad Ecológica resultante}

La calidad ecológica resultante queda determinada por el peor de los valores obtenidos en los indicadores ( $c f .$, DMA, ANEXO V). En el procedimiento propuesto para su determinación (ACA, 2006), se parte del valor obtenido mediante los indicadores biológicos, y se ajusta mediante la calidad fisicoquímica obtenida.

\subsection{Evaluación medioambiental del conjunto de presiones antrópicas}

2.3.1. Principales usos y actividades desarrolladas

Se analizan aquellos usos y actividades que se desarrollen dentro del ámbito de estudio, caracterizándolos con el máximo grado de detalle posible. 


\subsubsection{Identificación de impactos: Matriz de impactos}

Los impactos causados por cada actividad identificada, entendidos como una presión sobre el estado ecológico de la masa de agua, se identifican mediante una matriz de Impactos, inspirada en la matriz de Leopold (LEOPOLD et al., 1971). La nueva matriz difiere en que los factores ambientales considerados se ajustan a los criterios de la DMA (cf., DMA, ANEXO V).

Así, se estructuran en filas los factores ambientales y en columnas las presiones (Tabla 2). La matriz resultante representa todos los posibles impactos que estas presiones pueden causar sobre los diferentes factores ambientales.

Tabla 2. Correlación existente entre los distintos indicadores empleados para la determinación de la calidad ecológica y la caracterización del atributo Intensidad.

Table 2. Correlation between the different indicators used to measure the ecological quality and the intensity variable.

\begin{tabular}{|c|c|c|c|c|c|c|c|}
\hline \multicolumn{8}{|c|}{ INTENSIDAD } \\
\hline \multirow{2}{*}{\multicolumn{2}{|c|}{$\begin{array}{c}\text { FACTORES } \\
\text { AMBIENTALES }\end{array}$}} & \multirow{2}{*}{$\begin{array}{l}\text { MÉTODO } \\
\text { EMPLEADO PARA } \\
\text { SU VALORACIÓN }\end{array}$} & \multicolumn{5}{|c|}{$\begin{array}{c}\text { EQUIVALENCIA } \\
\text { CALIDAD \& INTENSIDAD }\end{array}$} \\
\hline & & & $\begin{array}{c}\text { Muy } \\
\text { Buena }\end{array}$ & Buena & Aceptable & Deficiente & Mala \\
\hline \multirow{4}{*}{ 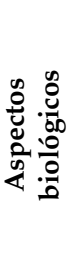 } & Flora acuática & Calidad Biológica & \multirow{2}{*}{\multicolumn{2}{|c|}{ Mínima }} & Media & Alta & Total \\
\hline & Fitoplancton & Final & & & & & \\
\hline & $\begin{array}{l}\text { Fauna } \\
\text { ictiológica }\end{array}$ & Presencia/Ausencia & \multicolumn{2}{|c|}{ Mínima } & Media & Alta & Total \\
\hline & $\begin{array}{l}\text { Fauna } \\
\text { bentónica de } \\
\text { invertebrados }\end{array}$ & IBMWP/InMacro & \multicolumn{3}{|c|}{ Ausencia $=$ Mínima } & \multicolumn{2}{|c|}{ Presencia $=$ Total } \\
\hline \multicolumn{2}{|c|}{$\begin{array}{c}\text { Aspectos } \\
\text { hidromorfológicos }\end{array}$} & $\begin{array}{c}\text { Calidad Ecológica } \\
\text { Final }\end{array}$ & \multicolumn{2}{|c|}{ Mínima } & Media & Alta & Total \\
\hline \multicolumn{2}{|c|}{$\begin{array}{c}\text { Aspectos } \\
\text { Fisicoquímicos }\end{array}$} & $\begin{array}{c}\text { Calidad Fisicoquímica } \\
\text { Final }\end{array}$ & \multicolumn{2}{|c|}{ Mínima } & Media & Alta & Total \\
\hline
\end{tabular}

La intensidad se define como una manifestación de la desviación respecto a un buen estado ecológico. Para su cálculo se utilizan los indicadores previamente evaluados para la determinación de la calidad ecológica. En la tabla se observan los distintos métodos empleados para cada factor ambiental y su equivalencia con el atributo intensidad. 
2.3.3. Evaluación de los impactos: matriz de importancia y panel de expertos

\section{- Matriz de importancia:}

La importancia de cada impacto se determina mediante una matriz de importancia (Conesa, 1996) adaptada a las características propias de estos entornos (cf., ARRUEBO et al., 2006; RODRÍGUEZ et al., 2009; Tabla 3).

Para su cálculo se emplean 10 atributos, que adquieren un valor en función de la caracterización que se le da, y son:

- Intensidad (I): indica el grado de incidencia de la acción sobre cada factor ambiental. Para ajustarlo a la DMA, se ha definido este atributo como una manifestación de la desviación respecto al estado de referencia (i.e., buen estado). De este modo, para cada uno de los factores ambientales, la Intensidad quedará valorada como Mínima (1), si el indicador que lo evalúa muestra una buena calidad, Media (4), si muestra una calidad aceptable, Alta (8), si ésta es deficiente, y Total (12), si es mala. Aquellos factores que no cuentan con un indicador específico para su evaluación, se valorarán considerando tanto las calidades finales resultantes (i.e., calidad biológica y fisicoquímica finales) como la observación in situ (Tabla 2).

- Extensión (EX): define el porcentaje de superficie respecto al total en que se manifiesta el efecto. Se considera Puntual (1), si el área afectada es inferior al 25\% de la total, Parcial (2), si ésta se encuentra entre el 25$50 \%$, Extenso (4), si supone entre 50-75\%, o Total (8), si es superior al $75 \%$. Además, la valoración anterior se verá incrementada en 4 unidades cuando la localización se considere crítica (i.e., situado dentro de los límites de un Espacio Natural Protegido).

- Momento (MO): valora el tiempo que transcurre entre la aparición de la acción y el comienzo del efecto sobre el factor considerado. Variará desde Inmediato (4), si el tiempo transcurrido es nulo, Medio Plazo (2), si es inferior a cinco años, hasta Largo Plazo (1), si transcurren más de cinco años. Por otra parte, si el momento del impacto fuese crítico (i.e., ocurre durante la época del año en la que no existe banquisa de hielo), el valor se incrementará en 4 unidades.

- Persistencia (PE): determina el tiempo que permanece el efecto desde su aparición hasta el momento en el que el factor afectado retorne a sus condiciones iniciales, bien por medios naturales o mediante la aplicación de medidas correctoras. Varía entre Fugaz (1), si es inferior a 1 año, Temporal (2), si se extiende entre 1 y 10 años, y Permanente, para los casos en que persista el efecto más de 10 años. 
Tabla 3. Matriz de impactos de Sabocos, con sus correspondientes valoraciones. Table 3. Matrix of impacts in Sabocos.

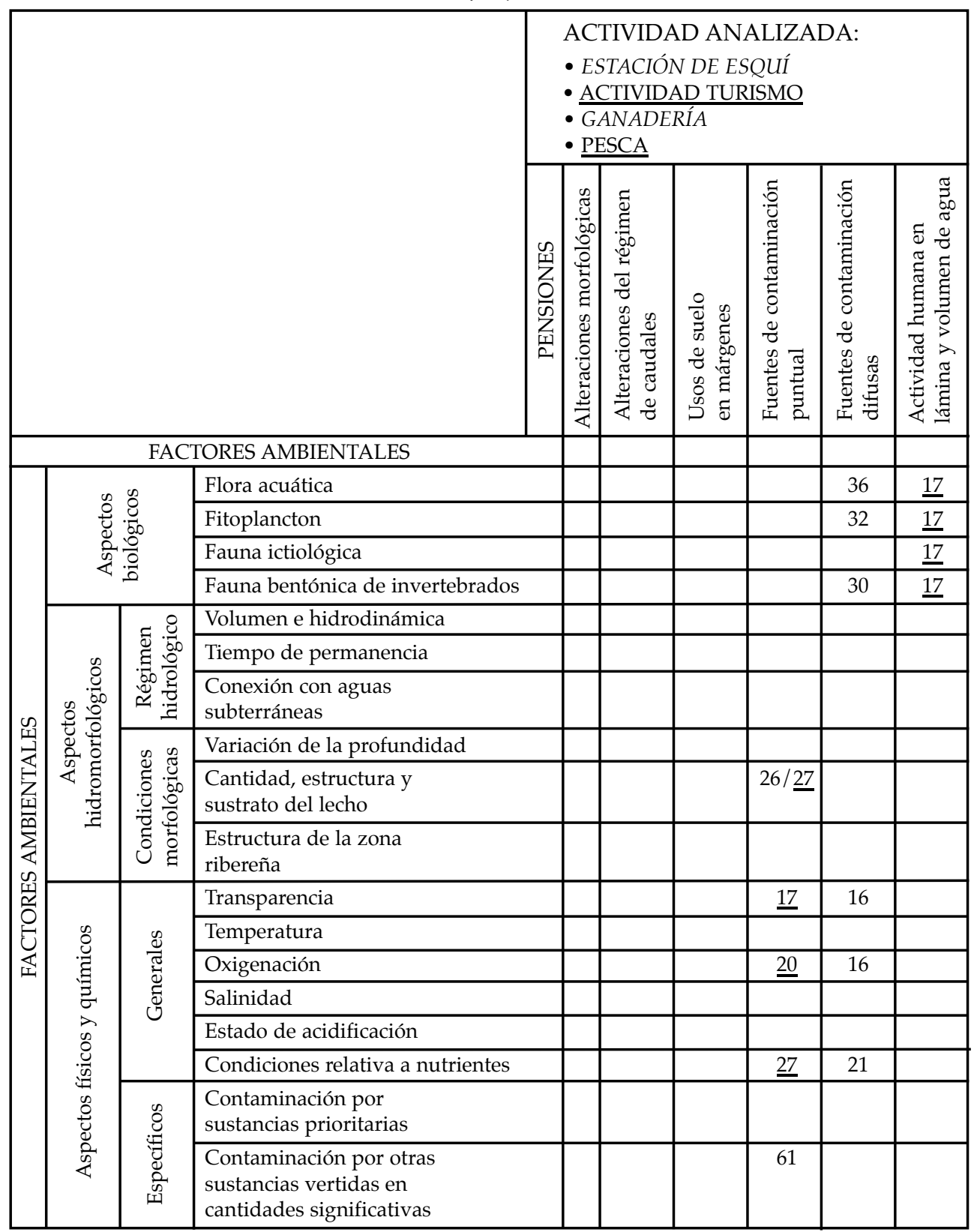

En la tabla se observan los principales impactos identificados con su correspondiente valoración. Se puede observar como los impactos más significativos derivan de la estación de esquí, el turismo y la ganadería, mientras que los menos relevantes provienen de la pesca. 
- Reversibilidad (RV): penaliza aquel impacto en el que el factor afectado no puede retornar a las condiciones iniciales de forma natural una vez que cesa la acción que lo provoca. La reversibilidad puede considerarse a Corto Plazo (1), si es inferior a 1 año, a Medio Plazo (2), si es entre 1 y 10 años, o Irreversible (4), si es superior a los 10 años.

- Recuperabilidad (MC): evalúa la posibilidad de reconstruir total o parcialmente las condiciones iniciales de forma artificial. Se valora con un (1) si es Totalmente Recuperable e Inmediato (i.e., en un periodo de tiempo inferior a 1 año), con un (2) si es Totalmente Recuperable a Medio Plazo (i.e., entre 1 y 10 años), con un (4) cuando es Mitigable (i.e., Parcialmente Recuperable en un plazo de tiempo inferior a 10 años), o con un (8) si es Irrecuperable (i.e., No Recuperable en un plazo de tiempo superior a 10 años).

- Sinergia (SI): muestra la posibilidad de que la componente final de la manifestación de dos o más efectos simples, provenientes de acciones simultáneas, sea superior a la que cabría esperar si estas acciones sucedieran en distinto momento temporal. Adquiere el valor (1) cuando las acciones son No Sinérgicas y (4) si presentan Sinergia.

- Acumulación (AC): determina el incremento progresivo de la manifestación de un efecto, cuando éste persiste de un modo continuado o cuando se lleva a cabo de una forma reiterada la acción que lo provoca. Si es una acción No Acumulativa, adquiere un valor de (1), y si es Acumulativa, se incrementa a (4).

- Efecto (EF): hace alusión al modo de manifestación de un efecto sobre un factor, a consecuencia de la acción. Será Directo (4), si la repercusión de la acción es consecuencia directa de ésta, o será Indirecto (1), si no lo es.

- Periodicidad (PR): mide la regularidad de la manifestación del efecto. Si es impredecible, se considerará Efecto Irregular (1), si es cíclica será un Efecto Periódico (2); o si es constante será un Efecto Continuo (4).

La Importancia de cada impacto se calcula mediante la expresión (CONESA, 1996):

* IMPORTANCIA = +/- (3·I + 2·EX + MO + PE + RV + SI + AC + EF $+\mathrm{PR}+\mathrm{MC})$

- Caracterización del impacto:

A fin de justificar las valoraciones consideradas para cada impacto, se procede a la caracterización de los impactos de forma sucinta y concreta, haciendo una especial alusión a los 10 atributos evaluados. 
- Panel de expertos:

Posteriormente, es necesario contrastar los resultados relativos a aquellos impactos más significativos y jerarquizarlos. Para ello, un panel de expertos valora cualitativamente y jerarquiza, de forma independiente, aquellos impactos que han resultado ser significativos mediante un cuestionario, previamente validado y fundamentado en el método de clasificación por grados escalares (GÓMEZ OREA, 2003). Los criterios de valoración y las nomenclaturas empleadas son:

a) Magnitud de la severidad (importancia) con la que se manifiesta el impacto (Tabla 4):

Tabla 4. Nomenclaturas y valor de referencia para la caracterización de la Importancia. Table 4. Names and reference values to characterise the Importance.

\begin{tabular}{|ccc|}
\hline \multicolumn{3}{|c|}{ IMPORTANCIA DEL IMPACTO } \\
\hline Compatibles (irrelevantes) & $\mathrm{CO}$ & 25 \\
Moderados & $\mathrm{MO}$ & 50 \\
Severos & $\mathrm{SE}$ & 75 \\
Críticos & $\mathrm{CR}$ & 100 \\
\hline
\end{tabular}

- Compatible (CO): la recuperación es inmediata tras el cese de la actividad, y no precisa prácticas protectoras o correctoras.

- Moderado (MO): la recuperación precisa medidas protectoras o correctoras de fácil aplicación y la restitución de las condiciones ambientales iniciales requiere cierto tiempo (i.e., inferior a 1 año).

- Severo (SE): la recuperación de las condiciones del medio exige la realización de medidas protectoras o correctoras de difícil aplicación o, aplicando medidas sencillas, precisa de un período de tiempo dilatado para su recuperación (i.e., superior a 1 año).

- Crítico (CR): cuando se produce una pérdida permanente de la calidad de las condiciones ambientales, sin posible recuperación, incluso con la adopción de medidas protectoras o correctoras.

b) Su repercusión sobre la calidad ecológica del ecosistema (Tabla 5):

- Incidencia Despreciable (ID): la manifestación no supone una alteración significativa de la calidad ecológica de las aguas.

- Incidencia Moderada (IM): Aquel que incide sobre la calidad ecológica, si bien sus consecuencias no exceden los umbrales de calidad ecológica aceptable ( $c f .$, DMA). 
T. ARRUEBO, A. PARDO, C. RODRÍGUEZ, F. J. LANAJA \& J. DEL VALLE

Tabla 5. Nomenclaturas y valor de referencia para la caracterización de la Repercusión. Table 5. Names and reference values to characterise the repercussion.

\begin{tabular}{|ccc|}
\hline \multicolumn{2}{|c|}{ REPERCUSIÓN SOBRE LA CALIDAD ECOLÓGICA } & \\
\hline Incidencia despreciable & ID & 25 \\
Incidencia moderada & IM & 50 \\
Incidencia severa & IS & 75 \\
Incidencia crítica & IC & 100 \\
\hline
\end{tabular}

- Incidencia Severa (IS): Aquel que incide sobre la calidad ecológica del lago amenazando los límites de calidad aceptable ( $c f .$, DMA). Exige la adecuación de un plan de medidas correctoras preciso para garantizar la integridad del ecosistema.

- Incidencia Crítica (IC): Aquel cuyas consecuencias pueden exceder los límites de calidad ecológica aceptable ( $c f .$, DMA). Con él se produce una pérdida permanente en la calidad, aún con la implantación de un plan de medidas correctoras.

c) Posibilidad de retornar al estado natural o mitigar sus consecuencias (Tabla 6):

Tabla 6. Nomenclaturas y valor de referencia para la caracterización de la Retornabilidad. Table 6. Names and reference values to characterise the recovering.

\begin{tabular}{|ccc|}
\hline \multicolumn{2}{|c|}{ RETORNABILIDAD } & \\
\hline Totalmente retornable & RT & 25 \\
Retornable a medio plazo & RM & 50 \\
Parcialmente retornable & RP & 75 \\
No retornable & NR & 100 \\
\hline
\end{tabular}

- Totalmente Retornable (RT): se puede retornar a las condiciones iniciales de un modo inmediato (i.e., inferior a 1 año).

- Retornable a Medio plazo (RM): si el tiempo necesario para retornar a las condiciones iniciales es superior a 1 año e inferior a 10 años.

- Parcialmente Retornable (RP): únicamente es posible corregir el impacto de un modo parcial en un tiempo inferior a 10 años.

- No Retornable (NR): es imposible corregir las consecuencias del impacto en un tiempo inferior a 10 años. 
Posteriormente, se pide a los panelistas la propuesta de aquellas medidas correctoras que crean oportunas, junto con la valoración de su aplicabilidad. Para ello, se han establecido los siguientes criterios y nomenclaturas:

- Inviable (I): debido el estado del arte o las condiciones económicas del momento, resulta inviable aplicar la medida correctora propuesta en un tiempo razonable.

- Muy Difícil (MD): existe una gran dificultad para aplicar dicha medida, bien por las características propias del lugar o bien por las limitaciones técnicas o económicas que ello conlleva.

- Difícil (D): las características propias del lugar, los aspectos técnicos o los recursos necesarios dificultan la aplicabilidad de dicha medida.

- Fácil (F): la aplicación de la medida conlleva unos recursos y conocimientos asequibles y relativamente sencillos, de acuerdo a las características del lugar.

- Muy Fácil (MF): la medida puede ser implantada de un modo muy sencillo y con pocos recursos.

En los supuestos en los que exista una discrepancia significativa entre los resultados de la matriz de importancia previa y la opinión de los panelistas, o entre ellos mismos, se reunirá a todos los miembros del panel para revisar las divergencias y consensuar una propuesta.

Elección de panelistas:

LARRY (1999) estableció los criterios que deben existir entre los distintos factores ambientales y los especialistas más indicados para evaluarlos. Siguiendo estas directrices, el panel deberá estar constituido, al menos, con profesionales especialistas de las siguientes áreas de conocimiento: biología, meteorología, geología, geografía y química.

\subsection{Interpretación de resultados: Matriz ICPA}

Los resultados obtenidos, una vez que han sido contrastados por el panel de expertos, y su interpretación se presentan cumplimentando una matriz que recoge el análisis de los impactos significativos, sus consecuencias y las medidas correctoras propuestas con una valoración de su aplicabilidad (matriz ICPA). La información que contiene cada campo de la matriz así como los criterios y nomenclaturas empleadas son los siguientes:

Respecto a los impactos se valora su repercusión sobre el estado ecológico de la masa de agua (REE) y su severidad e incidencia sobre las condiciones 
ambientales del lugar (Sev). Además, se incluye una breve descripción de los mismos. En ambos casos, la valoración vendrá reflejada en una escala jerárquica, cromática y numérica, clasificados desde el más relevante hasta el menos significante (Tabla 11).

A continuación, junto a la descripción de las consecuencias de cada impacto, se incluye la valoración sobre la retornabilidad, la propuesta de medidas correctoras y su aplicabilidad a partir de la información recopilada y contrastada por el panel de expertos. Para ello se siguen los mismos criterios y nomenclaturas propuestos al panel a la hora de valorar la posibilidad de retornar al estado natural o mitigar sus consecuencias y de la aplicabilidad de las medidas correctoras propuestas.

\section{Aplicación a un caso práctico: lago de Sabocos}

La base de datos completa obtenida durante el estudio realizado en el lago de Sabocos puede consultarse en RODRÍGUEZ et al. (2009). En este trabajo se resumen y destacan aquellos resultados más relevantes.

\subsection{Descripción del área de estudio: delimitación y caracterización de la masa de agua}

El lago de Sabocos, situado a $1905 \mathrm{~m}$ de altitud, corresponde a la red hidrográfica del río Gállego, dentro del valle de Tena, provincia de Huesca, municipio de Panticosa (Fig. 1), y se localiza dentro de los límites de la Reserva de la Biosfera Ordesa Viñamala. El lago de Sabocos se encuentra en la región biogeográfica Eurosiberiana, Provincia Pirenaica, Sector Pirenaico Central, subsector Altopirenaico (cf., RIVAS MARTÍNEZ, et al., 1991), se clasifica como Lago Alpino de Aguas Ácidas (AAM; ACA, 2006), y se encuentra localizado en la ecorregión Alta montaña según las ecorregiones de la cuenca del Ebro ( $c f$., PRAT y MUNNE, 1999).

Desde Huesca se accede a Panticosa a través de la N-330, tomando el desvío hacia la N-260 en Sabiñánigo y, una vez alcanzado el municipio de Biescas, por la carretera regional 136 que recorre el valle. Desde Panticosa salen sendas para ascender hasta la estación invernal de Panticosa. Éstas se unen cerca de la estación formando un único ramal. Previo a alcanzar el lago de los Asnos, a nuestra izquierda, hay un evidente desvío que conduce hasta el lago de Sabocos (Huso 30; x: 724.800, y: 4.730.500). 


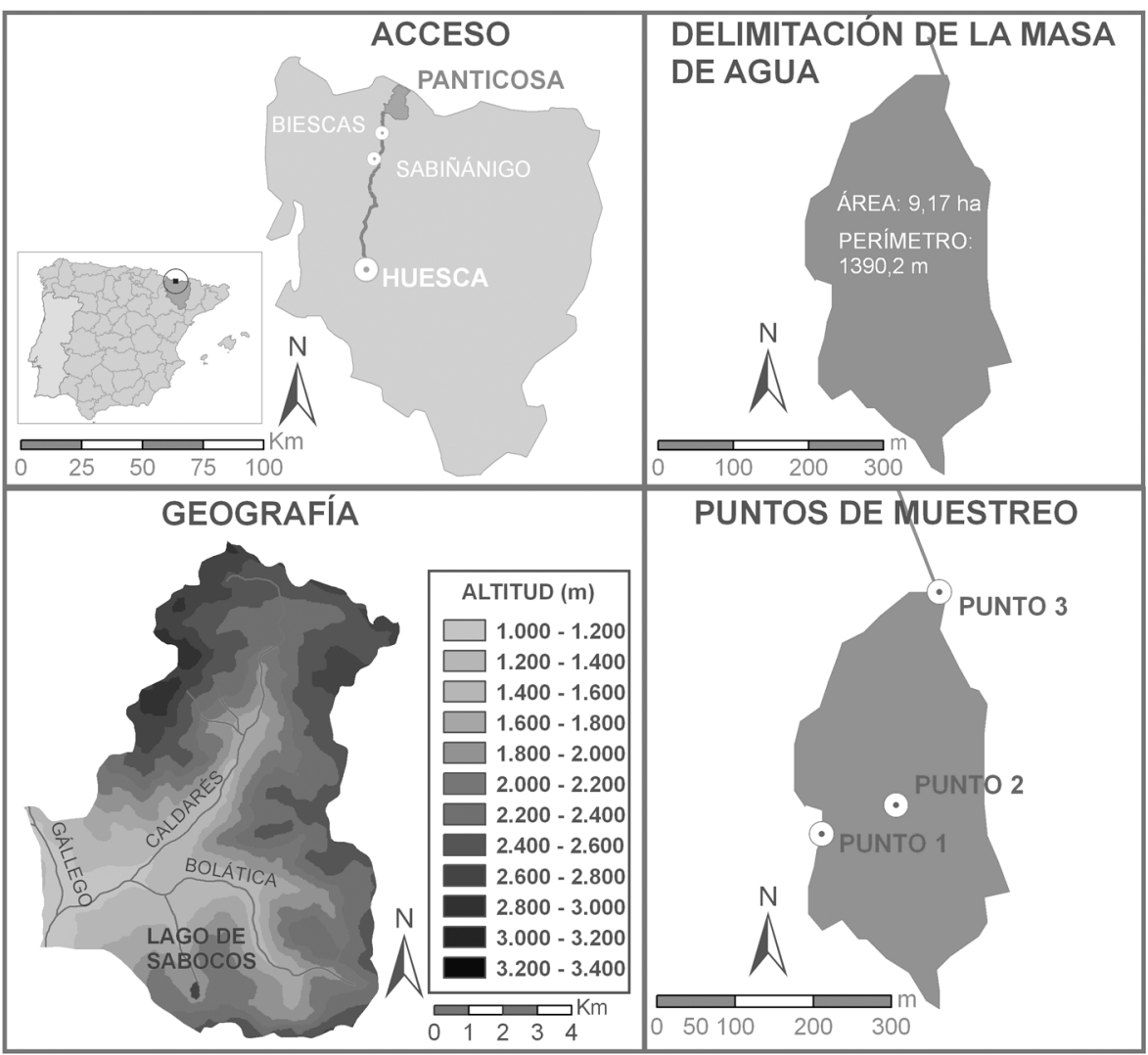

Figura 1. Acceso. Mapa de localización del municipio de Panticosa. Geografía. Red hidrográfica y descripción altitudinal del área de estudio. Delimitación de la masa de agua. Cota máxima alcanzada por la lámina de agua en el lago de Sabocos. Puntos de muestreo. Localización de los puntos muestreados para la determinación de la Calidad Ecológica.

Figure 1. Access: Situation map of Panticosa. Geography: Hydrographic net and altidudinal description of the study area. Limits of the water volume: Maximum altitude of the water sheet in the lake of Sabocos. Sampling points: Location of the sampling points.

\subsection{Evaluación de la situación actual del ecosistema: calidad ecológica}

\section{- Calidad biológica:}

El índice IBMWP y la presencia de peces son los principales parámetros determinantes de las calidades obtenidas (Tabla 7). 
El índice IBMWP se ha aplicado únicamente en el punto correspondiente a aguas lóticas (i.e., desagüe del lago, punto 3; Fig. 1). Se trata de una métrica ampliamente utilizada para la evaluación de torrentes de agua circulante (e.g., VAL, 2002; GARRIDO et al., 1998; MUÑOZ et al., 1998), siendo ésta su finalidad originaria (cf., Alba-Tercedor y Sanchez Ortega, 1988). Así, si bien únicamente se ha aplicado en aquellos puntos de aguas lóticas, su validez para este tipo de estudios en lagos debe ser, en principio, cuestionada. De hecho, si se correlacionan los resultados de este índice con InMacro, métrica específica para ecosistemas lacustres, se pueden observar diferencias notables (Tabla 7). Del mismo modo, si consideramos las familias identificadas (e.g., heptagenidae, perlodidae, potamanthidae, lepidostomatidae), se puede estimar una calidad biológica mejor que la definida por el índice IBMWP.

Tabla 7. Calidades biológicas alcanzadas. Table 7. Biological quality.

\begin{tabular}{|c|c|c|c|c|c|c|c|}
\hline \multirow[t]{2}{*}{ CAMPAÑA } & \multirow[t]{2}{*}{ PTO } & \multicolumn{2}{|c|}{ IBMWP } & \multicolumn{2}{|c|}{ InMacro } & \multirow{2}{*}{$\begin{array}{l}\text { Presencia } \\
\text { de peces }\end{array}$} & \multirow{2}{*}{$\begin{array}{l}\text { CALIDAD } \\
\text { BIOLÓGICA }\end{array}$} \\
\hline & & Resultado & Calidad & Resultado & Calidad & & \\
\hline Invierno & $\begin{array}{l}1 \\
2 \\
3\end{array}$ & $\begin{array}{l}- \\
- \\
17\end{array}$ & $\begin{array}{c}- \\
- \\
\text { Mala }\end{array}$ & 5 & $\begin{array}{l}\text { Muy } \\
\text { buena }\end{array}$ & $\begin{array}{l}\text { SI } \\
\text { SI } \\
\text { SI }\end{array}$ & $\begin{array}{c}\text { Buena } \\
\text { Buena } \\
\text { Mala }\end{array}$ \\
\hline Primavera & $\begin{array}{l}1 \\
2 \\
3\end{array}$ & $\begin{array}{c}- \\
- \\
33 \\
\end{array}$ & $\begin{array}{c}- \\
- \\
\text { Mala }\end{array}$ & 8 & $\begin{array}{l}\text { Muy } \\
\text { buena }\end{array}$ & $\begin{array}{l}\text { SI } \\
\text { SI } \\
\text { SI }\end{array}$ & $\begin{array}{c}\text { Buena } \\
\text { Buena } \\
\text { Mala }\end{array}$ \\
\hline Verano & $\begin{array}{l}1 \\
2 \\
3\end{array}$ & $\begin{array}{l}- \\
- \\
43\end{array}$ & $\begin{array}{c}- \\
- \\
\text { Escasa }\end{array}$ & 6 & $\begin{array}{l}\text { Muy } \\
\text { buena }\end{array}$ & $\begin{array}{l}\text { SI } \\
\text { SI } \\
\text { SI }\end{array}$ & $\begin{array}{c}\text { Buena } \\
\text { Buena } \\
\text { Deficiente }\end{array}$ \\
\hline Otoño & $\begin{array}{l}1 \\
2 \\
3\end{array}$ & $\begin{array}{l}- \\
- \\
24\end{array}$ & $\begin{array}{c}- \\
- \\
\text { Mala }\end{array}$ & 6 & $\begin{array}{l}\text { Muy } \\
\text { buena }\end{array}$ & $\begin{array}{l}\text { SI } \\
\text { SI } \\
\text { SI }\end{array}$ & $\begin{array}{c}\text { Buena } \\
\text { Buena } \\
\text { Mala }\end{array}$ \\
\hline
\end{tabular}

La calidad biológica alcanzada en los distintos muestreos ha sido Buena, excepto en el punto 3. Se puede observar como esta discrepancia deriva de la aplicación del índice IBMWP en este punto.

Durante las visitas continuadas al lago se ha identificado presencia de peces en sus aguas, lo que ha sido considerado como vector de afección (ACA, 2006). Históricamente se han introducido ejemplares de distintas especies para la pesca (e.g., Salmo trutta fario, Oncorhynchus mykiss) y, hoy en día, prácticamente en todos los lagos pirenaicos podemos encontrar alguna de estas especies. 
- Calidad fisicoquímica:

Los criterios expuestos por la CHE y por Adour-Garonne difieren significativamente, presentando distintos umbrales de calidad para algunos parámetros (e.g., $\mathrm{Ca}, \mathrm{Pb}, \mathrm{Cr})$. Así, las calidades fisicoquímicas determinadas a partir de ambas métricas son diferentes, resultando en la mayoría de los casos Adour-Garonne más restrictivo (Tabla 8).

Tabla 8. Calidades fisicoquímicas alcanzadas.

Table 8. Physical and Chemical quality.

\begin{tabular}{|c|c|c|c|c|c|c|}
\hline CAMPAÑA & PTOS & $\begin{array}{c}\text { Calidad } \\
\text { Teórica }\end{array}$ & $\begin{array}{c}\text { Adour- } \\
\text { Garonne }\end{array}$ & IPO & $\begin{array}{c}\text { LÍMITES } \\
\text { ACA }\end{array}$ & $\begin{array}{c}\text { CALIDAD } \\
\text { FISICOQUÍMICA }\end{array}$ \\
\hline Invierno & 1 & $\mathrm{C} 2$ & $\mathrm{C} 3$ & BAJA & $\mathrm{Sb}$ & Aceptable \\
& 2 & $\mathrm{C} 2$ & $\mathrm{C} 3$ & NULA & $\mathrm{Sb}$ & Aceptable \\
& 3 & $\mathrm{C} 4$ & $\mathrm{C} 3$ & $\mathrm{BAJA}$ & $\mathrm{Sb}$ & Deficiente \\
\hline Primavera & 1 & $\mathrm{C} 2$ & $\mathrm{C} 4$ & NULA & $\mathrm{Sb}$ & Deficiente \\
& 2 & $\mathrm{C} 2$ & $\mathrm{C} 5$ & NULA & $\mathrm{Sb}$ & Mala \\
& 3 & $\mathrm{C} 2$ & $\mathrm{C} 4$ & NULA & $\mathrm{Sb}$ & Deficiente \\
\hline Verano & 1 & $\mathrm{C} 4$ & $\mathrm{C} 5$ & NULA & $\mathrm{Sb}$ & Mala \\
& 2 & $\mathrm{C} 4$ & $\mathrm{C} 5$ & NULA & $\mathrm{Sb}$ & Mala \\
& 3 & $\mathrm{C} 4$ & $\mathrm{C} 5$ & NULA & $\mathrm{Sb}$ & Mala \\
\hline Otoño & 1 & $\mathrm{C} 4$ & $\mathrm{C} 5$ & NULA & $\mathrm{Sb}$ & Mala \\
& 2 & $\mathrm{C} 4$ & $\mathrm{C} 5$ & NULA & $\mathrm{Sb}$ & Mala \\
& 3 & $\mathrm{C} 4$ & $\mathrm{C} 4$ & NULA & $\mathrm{Sb}$ & Deficiente \\
\hline
\end{tabular}

Sb: sobrepasados.

La calidad fisicoquímica alcanzada en los distintos muestreos sigue un patrón aleatorio, siendo en la mayoría de los casos Mala. Se puede observar como Adour-Garonne es la métrica cuyos resultados han sido más desfavorables.

La métrica propuesta por ACA no presenta umbrales para las distintas categorías de calidades, únicamente unos valores límite exigibles (ACA, 2006). En todos los muestreos realizados los resultados analíticos han sobrepasado estos límites.

El índice IPO únicamente muestra una polución baja en la campaña de invierno, puntos 1 y 3 (Tabla 8). No obstante, los resultados analíticos muestran valores destacables de $\mathrm{DBO} 5 \mathrm{y} \mathrm{NH}_{4}{ }^{+}$, variables relacionadas con la carga orgánica de las aguas (Tabla 9). 
T. ARRUEBO, A. PARDO, C. RODRÍGUEZ, F. J. LANAJA \& J. DEL VALLE

Tabla 9. Principales parámetros limitantes de las calidades fisicoquímicas obtenidas. Table 9. Limiting parameters for physical and chemical qualities.

\begin{tabular}{|c|c|c|c|c|c|c|c|c|c|c|c|}
\hline ESTACIÓN & PTOS & $\begin{array}{c}\text { DBO5 } \\
\mathrm{mg} \mathrm{O}_{2} / 1\end{array}$ & $\begin{array}{c}\text { DQO } \\
\mathrm{mg} \mathrm{O}_{2} / 1\end{array}$ & $\begin{array}{c}\mathrm{O} 2 \\
\mathrm{mg} \mathrm{O}_{2} / 1\end{array}$ & $\begin{array}{l}\mathrm{NH}_{4}^{+} \\
\mathrm{mg} / 1\end{array}$ & $\begin{array}{c}\mathrm{Zn} \\
\mathrm{mg} / 1\end{array}$ & $\begin{array}{c}\mathrm{Pb} \\
\mathrm{mg} / 1\end{array}$ & $\begin{array}{c}\mathrm{Fe} \\
\mathrm{mg} / \mathrm{l}\end{array}$ & $\begin{array}{c}\mathrm{Cu} \\
\mathrm{mg} / 1\end{array}$ & $\left|\begin{array}{c}\mathrm{Hg} \\
\mathrm{mg} / 1\end{array}\right|$ & $\begin{array}{c}\mathrm{Cr} \\
\mathrm{mg} / 1\end{array}$ \\
\hline $\begin{array}{l}\text { Invierno } \\
\text { Invierno } \\
\text { Invierno }\end{array}$ & $\begin{array}{l}\text { Pto } 1 \\
\text { Pto } 2 \\
\text { Pto } 3\end{array}$ & $\begin{array}{l}4 \\
8\end{array}$ & & & $\begin{array}{l}0,32 \\
0,09\end{array}$ & $\begin{array}{l}0,08 \\
0,09 \\
0,09\end{array}$ & & & & & \\
\hline $\begin{array}{l}\text { Primavera } \\
\text { Primavera } \\
\text { Primavera }\end{array}$ & $\begin{array}{l}\text { Pto } 1 \\
\text { Pto } 2 \\
\text { Pto } 3\end{array}$ & & & $\begin{array}{l}7,3 \\
7,2\end{array}$ & $\begin{array}{c}0,07 \\
0,1\end{array}$ & & $\begin{array}{l}0,04 \\
0,05 \\
0,04\end{array}$ & $\begin{array}{l}0,22 \\
0,09\end{array}$ & 0,01 & & \\
\hline $\begin{array}{l}\text { Verano } \\
\text { Verano } \\
\text { Verano }\end{array}$ & $\begin{array}{l}\text { Pto } 1 \\
\text { Pto } 2 \\
\text { Pto } 3\end{array}$ & & & & & & $\begin{array}{l}0,05 \\
0,05 \\
0,19\end{array}$ & & & $\begin{array}{l}0,32 \\
0,14\end{array}$ & 0,32 \\
\hline $\begin{array}{l}\text { Otoño } \\
\text { Otoño } \\
\text { Otoño }\end{array}$ & $\begin{array}{l}\text { Pto } 1 \\
\text { Pto } 2 \\
\text { Pto } 3\end{array}$ & & $\begin{array}{l}42 \\
33\end{array}$ & & & & $\begin{array}{l}0,09 \\
0,05\end{array}$ & & & & 0,06 \\
\hline
\end{tabular}

La calidad ecológica final han sido Aceptable en la mayoría de los muestreos, excepto en el punto 3. La principal causa de estos resultados más desfavorables en la zona de desagüe es la aplicación del índice IBMWP en este punto.

La calidad fisicoquímica final es inferior a la esperada, principalmente debido a las concentraciones analizadas de $\mathrm{Zn}, \mathrm{Pb}, \mathrm{Cr}$ y $\mathrm{Hg}$ (Tabla 9). Los valores más elevados se analizaron durante la campaña de verano.

La aparición de los picos estacionales de $\mathrm{Zn}, \mathrm{Pb}$ y $\mathrm{Cr}$, coincide en el tiempo con las tareas de mantenimiento de la estación de esquí de Panticosa, por lo que podría inferirse que su origen estuviera en las torres de las telesillas y los trabajos de mantenimiento realizados en ellas.

Las pinturas anticorrosivas usadas en los postes contienen habitualmente $\mathrm{Pb}$ y $\mathrm{Cr}$. Una inspección ocular de los postes restaurados cercanos al lago (i.e., telesilla Corona de Aragón) indica que han sido decapados antes de ser pintados. Así, si los restos no fueron retirados convenientemente del entorno, la escorrentía terminaría por acumularlos en el fondo del lago, que es su cuenca sedimentaria natural.

Respecto al Zn, se trata de un elemento que se utiliza en estructuras de Fe como ánodo de sacrificio para evitar la corrosión. Esta protección con el tiempo se va descamando y por meteorización pudiera acabar en el lago de Sabocos.

Los valores analizados de $\mathrm{Hg}$ determinan la deposición atmosférica como vector de contaminación de este entorno. No obstante, el origen del mercurio ha de ser estudiado con detalle. Aplicar modelos de dispersión atmosférica, analizando posibles retrotrayectorias de éstos, sería una buena herramienta para conocer posibles fuentes de contaminación ( $c f$. ., VAN DROOGE, et al., 2002, MAST et al., 2003). 


\section{- Calidad Ecológica:}

La Calidad Ecológica determinada en el lago de Sabocos ha sido Aceptable en los puntos 1 y 2, y Mala en casi todos los muestreos realizados en el punto 3 (ver tabla 10). No obstante, si despreciamos los resultados del índice IBMWP, tal y como se ha comentado anteriormente, dicha calidad sería Aceptable en todos los puntos.

Tabla 10. Calidad Ecológica resultante.

Table 10. Summary of ecological quality.

\begin{tabular}{|c|c|c|c|c|}
\hline CAMPAÑA & PTOS & $\begin{array}{l}\text { CALIDAD } \\
\text { BIOLÓGICA }\end{array}$ & $\begin{array}{c}\text { CALIDAD } \\
\text { FISICOQUÍMICA }\end{array}$ & $\begin{array}{l}\text { CALIDAD } \\
\text { ECOLÓGICA }\end{array}$ \\
\hline Invierno & $\begin{array}{l}1 \\
2 \\
3\end{array}$ & $\begin{array}{l}\text { Buena } \\
\text { Buena } \\
\text { Mala }\end{array}$ & $\begin{array}{l}\text { Aceptable } \\
\text { Aceptable } \\
\text { Deficiente }\end{array}$ & $\begin{array}{c}\text { Aceptable } \\
\text { Aceptable } \\
\text { Mala }\end{array}$ \\
\hline Primavera & $\begin{array}{l}1 \\
2 \\
3\end{array}$ & $\begin{array}{l}\text { Buena } \\
\text { Buena } \\
\text { Mala }\end{array}$ & $\begin{array}{c}\text { Deficiente } \\
\text { Mala } \\
\text { Deficiente }\end{array}$ & $\begin{array}{c}\text { Aceptable } \\
\text { Aceptable } \\
\text { Mala }\end{array}$ \\
\hline Verano & $\begin{array}{l}1 \\
2 \\
3\end{array}$ & $\begin{array}{c}\text { Buena } \\
\text { Buena } \\
\text { Deficiente }\end{array}$ & $\begin{array}{l}\text { Mala } \\
\text { Mala } \\
\text { Mala }\end{array}$ & $\begin{array}{l}\text { Aceptable } \\
\text { Aceptable } \\
\text { Deficiente }\end{array}$ \\
\hline Otoño & $\begin{array}{l}1 \\
2 \\
3\end{array}$ & $\begin{array}{c}\text { Buena } \\
\text { Buena } \\
\text { Mala }\end{array}$ & $\begin{array}{c}\text { Mala } \\
\text { Mala } \\
\text { Deficiente }\end{array}$ & $\begin{array}{c}\text { Aceptable } \\
\text { Aceptable } \\
\text { Mala }\end{array}$ \\
\hline \multicolumn{4}{|c|}{ CALIDAD ECOLÓGICA FINAL } & MALA \\
\hline
\end{tabular}

\subsection{Evaluación medioambiental del conjunto de presiones antrópicas}

\subsubsection{Principales usos y actividades desarrolladas}

Las principales actividades que pueden afectar al lago de Sabocos son:

- Estación de esquí de Panticosa: es gestionada por la sociedad holding Aramon, Montañas de Aragón, S.A., y sus $34 \mathrm{~km}$ esquiables se localizan en un entorno próximo al lago. En la temporada 2004/2005, la afluencia de público a esta estación se cifró en cerca de 70.000 esquiadores.

- Actividad turística: los turistas son escasos, excepto en el periodo estival. Durante los meses de julio y agosto, desde la estación de esquí se facilita su acceso mediante una telecabina. El balance estival del año 2005 se cifró en 22.000 personas. Se contabilizó el número de practi- 
cantes durante estos meses y se consultó a visitantes asiduos. Así, se ha estimado en unos 11.000 (i.e., $50 \%$ del total) el número de turistas que visitaron el lago de Sabocos.

- Ganadería: la actividad ganadera de Panticosa se limita fundamentalmente a ganado bovino y ovino. El número de cabezas censadas en el año 1999 eran 895 y 1.238 respectivamente (cf., IAEST, 2007). Durante las visitas periódicas se ha observado, en distintas ocasiones, ganado vacuno en las proximidades -40-50 cabezas-. También son observables excrementos en el perímetro durante el periodo estival, lo que denota la presencia estacional de este tipo de ganado.

- Actividades subacuáticas: en Sabocos se desarrollan puntualmente dos modalidades de buceo: en altitud durante el periodo estival y bajo hielo durante los meses en los que permanece cubierto bajo una banquisa de hielo. La Federación Aragonesa de Actividades Subacuáticas (FARAS) destaca la dificultad de acceso como factor limitante del número de practicantes. Hasta el año 2005, si bien no hay datos contrastados, se conoce la presencia esporádica de unos pocos buceadores en esta agua.

- Pesca: toda especie piscícola que habita en estos lagos se considerada introducida, puesto que sus condiciones de aislamiento impiden su acceso de un modo natural. No obstante, si bien no se han observado pescadores durante las visitas, si que son visibles peces desde sus orillas. Del mismo modo, los habitantes del lugar señalan que es habitual la práctica de esta disciplina.

\subsubsection{Identificación de impactos: Matriz de impactos}

Los principales impactos identificados se muestran en la tabla 3.

3.3.3. Evaluación de los impactos: matriz de importancia y panel de expertos

Las valoraciones que se les ha dado a cada uno de estos impactos se muestran en la tabla 3. Su caracterización ha sido:

- Estación de esquí

- Fuente de contaminación puntual - Cantidad, estructura y sustrato del lecho. Se han observado en el lecho utensilios para la delimitación de pistas (i.e.., palos, balizas) y residuos provenientes de esquiadores (e.g., envoltorios, bolsas de plástico, latas). 
MÉTODO ESPECÍFICO PARA LA EVALUACIÓN MEDIOAMBIENTAL DE LOS LAGOS DE ORIGEN...

\begin{tabular}{|c|c|c|c|c|}
\hline Signo & - & Recuperabilidad & 1 & \multirow{5}{*}{$\begin{array}{l}\text { La intensidad es mínima, al ser escasos los } \\
\text { residuos identificados en el fondo del lago. } \\
\text { El volumen de agua afectado es inferior al } \\
25 \% \text { del total, si bien se localiza dentro de la } \\
\text { Reserva de la Biosfera Ordesa Viñamala. El } \\
\text { tiempo transcurrido desde la aparición de la } \\
\text { acción y la manifestación de sus efectos es } \\
\text { nulo. Rápidamente recuperable, si bien } \\
\text { requiere intervención humana. Siendo direc- } \\
\text { to y periódico, no presenta sinergia, pero sus } \\
\text { efectos si que se incrementan progresiva- } \\
\text { mente si persiste la actividad. }\end{array}$} \\
\hline Intensidad & 1 & Sinergia & 1 & \\
\hline Extensión & 5 & Acumulación & 4 & \\
\hline Momento & 4 & Efecto & 4 & \\
\hline Persistencia & 1 & Periodicidad & 2 & \\
\hline Reversibilidad & 4 & IMPORTANCIA & 34 & \\
\hline
\end{tabular}

- Fuente de contaminación difusa - características fisicoquímicas. La calidad fisicoquímica obtenida ha sido Mala en la mayoría de los muestreos. El origen de algunas afecciones detectadas (i.e., [Cr], [Zn] y [Pb]) podrían derivar del mantenimiento de la estación.

\begin{tabular}{|c|c|c|c|c|}
\hline Signo & - & Recuperabilidad & 1 & \multirow{6}{*}{$\begin{array}{l}\text { La intensidad es alta, al ser mala la calidad } \\
\text { fisicoquímica obtenida en la mayoría de los } \\
\text { muestreos. Todo el lago se ve afectado y } \\
\text { transcurren varias semanas entre la acción y } \\
\text { el comienzo de sus efectos. Reversible a corto } \\
\text { plazo si cesa la actividad, es directo y perió- } \\
\text { dico, reapareciendo siempre que se realizan } \\
\text { estas tareas de mantenimiento. No siendo } \\
\text { sinérgico, sus efectos se incrementan progre- } \\
\text { sivamente si persiste la actividad. }\end{array}$} \\
\hline Intensidad & 8 & Sinergia & 1 & \\
\hline Extensión & 12 & Acumulación & 4 & \\
\hline Momento & 8 & Efecto & 4 & \\
\hline Persistencia & 1 & Periodicidad & 2 & \\
\hline Reversibilidad & 4 & IMPORTANCIA & 73 & \\
\hline
\end{tabular}




\section{- Actividad Turística}

- Fuente de contaminación puntual - Cantidad, estructura y sustrato del lecho. Se han recogido desperdicios que denotan la presencia de turistas (e.g., latas, plásticos, fragmentos de vidrio).

\begin{tabular}{|c|c|c|c|c|}
\hline Signo & - & Recuperabilidad & 1 & \multirow{6}{*}{$\begin{array}{l}\text { La intensidad es mínima pues, si bien son } \\
\text { abundantes los residuos que se observan, } \\
\text { únicamente unos pocos terminan en el } \\
\text { fondo. Al ser residuos sólidos, el tiempo } \\
\text { transcurrido desde la aparición de la acción y } \\
\text { el comienzo de sus efectos es nulo, y sería } \\
\text { inmediatamente recuperable si se limpiase el } \\
\text { lago. Es una acción directa y continua. A } \\
\text { pesar de no ser sinérgica, sus efectos se incre- } \\
\text { mentan progresivamente si se siguen deposi- } \\
\text { tando residuos sin control. }\end{array}$} \\
\hline Intensidad & 1 & Sinergia & 1 & \\
\hline Extensión & 5 & Acumulación & 4 & \\
\hline Momento & 8 & Efecto & 4 & \\
\hline Persistencia & 1 & Periodicidad & 2 & \\
\hline Reversibilidad & 4 & IMPORTANCIA & 39 & \\
\hline
\end{tabular}

- Fuente de contaminación puntual - Condición relativa a nutrientes. La deposición de residuos orgánicos por los turistas incrementa la carga orgánica de las aguas.

\begin{tabular}{|c|c|c|c|c|}
\hline Signo & - & Recuperabilidad & 1 & \multirow{6}{*}{$\begin{array}{l}\text { La polución orgánica analizada es nula en la } \\
\text { mayoría de los muestreos. No obstante, } \\
\text { habiéndose analizado concentraciones des- } \\
\text { tacables de algunos nutrientes, se ha consi- } \\
\text { derado de intensidad media. Sería reversible } \\
\text { a corto plazo si cesase la actividad. La mani- } \\
\text { festación directa es un incremento de mate- } \\
\text { ria orgánica. Su aparición conlleva un perio- } \\
\text { do breve y es sinérgico, pues la ganadería } \\
\text { también incrementa la carga orgánica. Los } \\
\text { nitratos se pueden fijar en forma de materia } \\
\text { vegetal. }\end{array}$} \\
\hline Intensidad & 2 & Sinergia & 4 & \\
\hline Extensión & 5 & Acumulación & 4 & \\
\hline Momento & 5 & Efecto & 4 & \\
\hline Persistencia & 1 & Periodicidad & 4 & \\
\hline Reversibilidad & 1 & IMPORTANCIA & 41 & \\
\hline
\end{tabular}


- Ganadería

- Fuente de contaminación difusa - Flora acuática. Las heces derivadas del ganado estacional es una fuente de eutrofización, incrementando la carga orgánica y afectando a la biota acuática.

\begin{tabular}{|c|c|c|c|c|}
\hline Signo & - & Recuperabilidad & 2 & \multirow{5}{*}{$\begin{array}{l}\text { No se han utilizado índices para valorar la } \\
\text { flora acuática, pero la observación in situ no } \\
\text { muestra indicios de alteraciones. Por ello, } \\
\text { esta afección se considera mínima. Siendo } \\
\text { leve, afecta a todo el lago. Es indirecta, pues } \\
\text { es consecuencia del incremento de nutrien- } \\
\text { tes, y el tiempo transcurrido desde la presen- } \\
\text { cia de ganado y la alteración de la biota es de } \\
\text { varias semanas. Si cesase esta actividad esta- } \\
\text { cional, el sistema tardaría más de un año a } \\
\text { retornar a su estado inicial de un modo natu- } \\
\text { ral. Nitratos y fosfatos pueden ser acumula- } \\
\text { dos en forma de materia vegetal. }\end{array}$} \\
\hline Intensidad & 1 & Sinergia & 1 & \\
\hline Extensión & 12 & Acumulación & 4 & \\
\hline Momento & 6 & Efecto & 1 & \\
\hline Persistencia & 2 & Periodicidad & 2 & \\
\hline Reversibilidad & 2 & IMPORTANCIA & 48 & \\
\hline
\end{tabular}

- Fuente de contaminación difusa - Fitoplancton. Las heces derivadas del ganado estacional es una fuente de eutrofización, incrementando la carga orgánica y afectando al fitoplancton.

\begin{tabular}{|c|c|c|c|c|}
\hline Signo & - & Recuperabilidad & 2 & \multirow{5}{*}{$\begin{array}{l}\text { No se han utilizado índices para valorar e } \\
\text { fitoplancton. Apoyados en la ausencia de } \\
\text { eutrofia y la nula turbidez de las aguas, } \\
\text { podemos predecir una afección mínima. E } \\
\text { fitoplancton se extiende en la columna de } \\
\text { agua de un modo estratificado. La afección } \\
\text { directa y a medio plazo son cambios en e } \\
\text { estado trófico, lo que altera las comunidades } \\
\text { fitoplanctónicas. Si cesase esta actividad } \\
\text { estacional, de forma natural recuperaría su } \\
\text { estado inicial en un periodo corto de tiempo } \\
\text { Así, nitratos y fosfatos pueden ser acumula- } \\
\text { dos en forma de materia vegetal. }\end{array}$} \\
\hline Intensidad & 1 & Sinergia & 1 & \\
\hline Extensión & 12 & Acumulación & 4 & \\
\hline Momento & 6 & Efecto & 1 & \\
\hline Persistencia & 1 & Periodicidad & 2 & \\
\hline Reversibilidad & 1 & IMPORTANCIA & 44 & \\
\hline
\end{tabular}


- Fuente de contaminación difusa - Fauna bentónica de invertebrados. La presencia de ganado vacuno provoca un incremento de la polución orgánica, que varía el estado trófico y puede alterar las comunidades bentónicas que habitan en lecho.

\begin{tabular}{|c|c|c|c|c|}
\hline Signo & - & Recuperabilidad & 2 & \multirow{5}{*}{$\begin{array}{l}\text { InMacro refleja un buen estado de las comu- } \\
\text { nidades bentónicas del conjunto del lago, por } \\
\text { lo que la intensidad es mínima y su extensión } \\
\text { generalizada. Aparece a medio plazo, pues } \\
\text { son organismos con un ciclo de vida entre un } \\
\text { mes y más de un año. Las comunidades ben- } \\
\text { tónicas se ven afectadas indirectamente tras } \\
\text { cambiar el estado trófico, y si cesase esta acti- } \\
\text { vidad periódica, el sistema tardaría en retor- } \\
\text { nar a las condiciones iniciales de modo natu- } \\
\text { ral más de un año. No es acumulativo, pues } \\
\text { los organismos únicamente se adaptan al } \\
\text { estado trófico presente. }\end{array}$} \\
\hline Intensidad & 1 & Sinergia & 1 & \\
\hline Extensión & 12 & Acumulación & 1 & \\
\hline Momento & 6 & Efecto & 1 & \\
\hline Persistencia & 2 & Periodicidad & 2 & \\
\hline Reversibilidad & 2 & IMPORTANCIA & 42 & \\
\hline
\end{tabular}

- Panel de expertos:

Los integrantes del panel de expertos han sido:

- José Espona: Lcdo. en Ciencias Biológicas y auditor de Sistemas de Gestión Medioambiental (acreditación IRCA, Norma UNE-EN ISO 19011).

- Evelio Álvarez: Lcdo. en Ciencias Físicas y Jefe GPV del Centro Meteorológico Territorial en Aragón, La Rioja y Navarra.

- Javier del Valle: Dr. en Geografía, profesor ayudante del Dpto. de Geografía y Ordenación del Territorio de la Univ. de Zaragoza y consultor técnico de la CHE.

- José María Matesanz: Dr. en Ciencias Químicas y profesor ayudante del Dpto. de Ingeniería Química y Tecnologías del Medio Ambiente de la Univ. de Zaragoza.

Los impactos valorados se muestran en la tabla 11. 
MÉTODO ESPECÍFICO PARA LA EVALUACIÓN MEDIOAMBIENTAL DE LOS LAGOS DE ORIGEN...

Tabla 11. Impactos valorados.

Table 11. Observed impacts.

\begin{tabular}{|c|c|l|c|}
\hline \multicolumn{2}{|c|}{ LAGO DE SABOCOS } \\
\hline N. ${ }^{\text {IMPACTO }}$ & ACTIVIDAD & \multicolumn{1}{c|}{ DESCRIPCIÓN } \\
\hline 1 & GANADERÍA & $\begin{array}{l}\text { Las heces derivadas de la presencia estacional de ganado } \\
\text { vacuno se comporta como fuente de eutrofización de las } \\
\text { aguas, incrementando la carga orgánica que llega y afectando } \\
\text { a la biota subacuática. }\end{array}$ \\
\hline 2 & TURISMO & $\begin{array}{l}\text { Residuos diversos provenientes de esta actividad (e.g., latas, } \\
\text { envoltorios, bolsas de plástico, colillas, etc), terminan deposi- } \\
\text { tados tanto en las orillas como en el fondo del lago. }\end{array}$ \\
\hline 3 & TURISMO & $\begin{array}{l}\text { Algunos de estos residuos son orgánicos. Su deposición final } \\
\text { en las aguas del lago deriva en un incremento de su carga } \\
\text { orgánica, afectando a las condiciones relativas a nutrientes. }\end{array}$ \\
\hline 4 & ESTACIÓN & $\begin{array}{l}\text { Se han observado utensilios empleados para la delimitación } \\
\text { de pistas en el fondo del lago (e.g., palos, balizas y otros). Del } \\
\text { mismo modo, residuos provenientes de la práctica de esta } \\
\text { actividad (e.g., envoltorios, bolsas de plástico, latas) terminan } \\
\text { depositados en su lecho. }\end{array}$ \\
\hline 5 & $\begin{array}{c}\text { ESTACIÓN } \\
\text { DE ESQUÍ }\end{array}$ & $\begin{array}{l}\text { Los materiales empleados para el mantenimiento de la esta- } \\
\text { ción (e.g., pinturas antioxidantes, vertidos de líquidos, resi- } \\
\text { duos varios) se comportan como fuente de contaminación } \\
\text { difusa, afectando directamente y de un modo significativo a } \\
\text { las propiedades fisicoquímicas de las aguas. }\end{array}$ \\
\hline
\end{tabular}

a) Magnitud de la severidad con la que se manifiesta cada uno de los impactos:

\begin{tabular}{|c|c|c|c|c|}
\hline \multirow{2}{*}{5 elementos } & \multicolumn{4}{|c|}{ Panelistas } \\
\cline { 2 - 5 } & $\mathbf{1}^{\mathbf{0}}$ & $\mathbf{2}^{\mathbf{o}}$ & $\mathbf{3}^{\mathbf{o}}$ & $\mathbf{4}^{\mathbf{o}}$ \\
\hline 1 & $\mathrm{CO}(25)$ & $\mathrm{CO}(25)$ & $\mathrm{CO}(25)$ & $\mathrm{SE}(75)$ \\
2 & $\mathrm{MO} \mathrm{(50)}$ & $\mathrm{MO}(50)$ & $\mathrm{MO}(50)$ & $\mathrm{MO}(50)$ \\
3 & $\mathrm{MO} \mathrm{(50)}$ & $\mathrm{CO}(25)$ & $\mathrm{CO}(25)$ & $\mathrm{CO}(25)$ \\
4 & $\mathrm{MO} \mathrm{(50)}$ & $\mathrm{MO}(50)$ & $\mathrm{MO}(50)$ & $\mathrm{MO}(50)$ \\
5 & $\mathrm{SE} \mathrm{(75)}$ & $\mathrm{MO}(50)$ & $\mathrm{SE}(75)$ & $\mathrm{SE}(75)$ \\
$\Sigma \mathrm{E}_{\mathrm{ei}}$ & 250 & 200 & 225 & 275 \\
\hline
\end{tabular}

\begin{tabular}{|c|c|c|c|c|c|c|}
\hline \multirow[t]{2}{*}{5 elementos } & \multicolumn{6}{|c|}{ Ponderación (Vei/ $\Sigma \mathrm{E}_{\mathrm{ei}}$} \\
\hline & $1^{\circ}$ & $2^{\circ}$ & $3^{\circ}$ & $4^{\circ}$ & $\sum$ Vei & Ve \\
\hline 1 & 0,100 & 0,125 & 0,111 & 0,273 & 0,609 & 0,152 \\
\hline 2 & 0,200 & 0,250 & 0,222 & 0,182 & 0,854 & 0,214 \\
\hline 3 & 0,200 & 0,125 & 0,111 & 0,091 & 0,527 & 0,132 \\
\hline 4 & 0,200 & 0,250 & 0,222 & 0,182 & 0,854 & 0,214 \\
\hline 5 & 0,300 & 0,250 & 0,333 & 0,273 & 1,156 & 0,289 \\
\hline & & & & $\sum \sum$ Vei & 4 & $(1)$ \\
\hline
\end{tabular}


T. ARRUEBO, A. PARDO, C. RODRÍGUEZ, F. J. LANAJA \& J. DEL VALLE

b) Su repercusión sobre la calidad ecológica:

\begin{tabular}{|c|c|c|c|c|}
\hline \multirow{2}{*}{5 elementos } & \multicolumn{4}{|c|}{ Panelistas } \\
\cline { 2 - 5 } & $\mathbf{1}^{\mathbf{o}}$ & $\mathbf{2}^{\mathbf{o}}$ & $\mathbf{3}^{\mathbf{o}}$ & $\mathbf{4}^{\mathbf{}}$ \\
\hline 1 & $\mathrm{IM}(50)$ & ID (25) & ID (25) & IS (75) \\
2 & $\mathrm{IM} \mathrm{(50)}$ & IM (50) & ID (25) & IM (50) \\
3 & IM (50) & ID (25) & ID (25) & ID (25) \\
4 & IS (75) & IM (50) & ID (25) & IM (50) \\
5 & IS (75) & IM (50) & IS (75) & IS (75) \\
$\sum \mathrm{E}_{\mathrm{ei}}$ & 300 & 200 & 175 & 275 \\
\hline
\end{tabular}

\begin{tabular}{|c|c|c|c|c|c|c|}
\hline \multirow{2}{*}{ 5 elementos } & \multicolumn{7}{|c|}{ Ponderación $\left(\right.$ Vei/ $/ \mathbf{E}_{\text {ei }}$} \\
\cline { 2 - 7 } & $\mathbf{1}^{\mathbf{o}}$ & $\mathbf{2}^{\mathbf{o}}$ & $\mathbf{3}^{\mathbf{o}}$ & $\mathbf{4}^{\mathbf{0}}$ & $\Sigma$ Vei & Ve \\
\hline 1 & 0,167 & 0,125 & 0,143 & 0,273 & 0,708 & 0,177 \\
2 & 0,167 & 0,250 & 0,143 & 0,182 & 0,742 & 0,186 \\
3 & 0,167 & 0,125 & 0,143 & 0,091 & 0,526 & 0,132 \\
4 & 0,250 & 0,250 & 0,143 & 0,182 & 0,825 & 0,206 \\
5 & 0,250 & 0,250 & 0,429 & 0,273 & 1,202 & 0,301 \\
& & & & $\sum \sum$ Vei & 4 & $(1)$ \\
\hline
\end{tabular}

c) Posibilidad de retornar a las condiciones naturales o mitigar sus consecuencias:

\begin{tabular}{|c|c|c|c|c|}
\hline \multirow[t]{2}{*}{5 elementos } & \multicolumn{4}{|c|}{ Panelistas } \\
\hline & $1^{\circ}$ & $2^{\circ}$ & $3^{\circ}$ & $4^{o}$ \\
\hline 1 & RM (50) & ID (25) & RT (25) & $\mathrm{RM}(50)$ \\
\hline 2 & RM (50) & RP (75) & RT (25) & RM (50) \\
\hline 3 & RM (50) & RT (25) & RT (25) & RT (25) \\
\hline 4 & RM (50) & RP (75) & RT (25) & RM (50) \\
\hline 5 & RP (75) & RM (50) & RM (50) & $\mathrm{RP}(75)$ \\
\hline$\sum \mathrm{E}_{\mathrm{ei}}$ & 3000 & 250 & 150 & 250 \\
\hline
\end{tabular}

\begin{tabular}{|c|c|c|c|c|c|c|}
\hline \multirow[t]{2}{*}{5 elementos } & \multicolumn{6}{|c|}{ Ponderación $\left(\right.$ Vei $/ \Sigma \mathrm{E}_{\mathrm{ei}}$} \\
\hline & $1^{o}$ & $2^{\circ}$ & $3^{\circ}$ & $4^{\circ}$ & $\sum$ Vei & Ve \\
\hline 1 & 0,182 & 0,100 & 0,167 & 0,200 & 0,649 & 0,162 \\
\hline 2 & 0,182 & 0,300 & 0,167 & 0,200 & 0,849 & 0,212 \\
\hline 3 & 0,182 & 0,100 & 0,167 & 0,100 & 0,549 & 0,137 \\
\hline 4 & 0,182 & 0,300 & 0,167 & 0,200 & 0,849 & 0,212 \\
\hline 5 & 0,273 & 0,200 & 0,333 & 0,300 & 1,106 & 0,277 \\
\hline & & & & $\sum \sum$ Vei & 4 & (1) \\
\hline
\end{tabular}


MÉTODO ESPECÍFICO PARA LA EVALUACIÓN MEDIOAMBIENTAL DE LOS LAGOS DE ORIGEN...

d) Medidas correctoras propuestas por los panelistas y valoración de su aplicabilidad:

\begin{tabular}{|c|c|c|c|c|c|c|c|c|}
\hline \multirow[t]{2}{*}{ Elementos } & \multicolumn{8}{|c|}{ Panelistas } \\
\hline & \multicolumn{2}{|l|}{$1^{\circ}$} & \multicolumn{2}{|l|}{$2^{\circ}$} & \multicolumn{2}{|l|}{$3^{\circ}$} & \multicolumn{2}{|l|}{$4^{\circ}$} \\
\hline 1 & $\begin{array}{l}\text { Controlar los } \\
\text { accesos para } \\
\text { el ganado }\end{array}$ & $\mathrm{F}$ & - & - & $\begin{array}{l}\text { Limitación } \\
\text { carga } \\
\text { ganadera en } \\
\text { la cuenca del } \\
\text { ibón }\end{array}$ & D & - & - \\
\hline 2 & \multirow[t]{3}{*}{$\begin{array}{l}\text { Educación, } \\
\text { concienciación }\end{array}$} & \multirow[t]{3}{*}{$\mathrm{F}$} & \multirow{3}{*}{$\begin{array}{l}\text { Señalización } \\
\text { de la zona } \\
\text { recordando } \\
\text { la necesidad } \\
\text { de preservar } \\
\text { el entorno }\end{array}$} & \multirow[t]{3}{*}{ MF } & \multirow{2}{*}{$\begin{array}{l}\text { Actividades de } \\
\text { educación } \\
\text { ambiental }\end{array}$} & \multirow{2}{*}{ F } & $\begin{array}{l}\text { Recogida de } \\
\text { residuos }\end{array}$ & $\mathrm{F}$ \\
\hline 3 & & & & & & & $\begin{array}{l}\text { Regulación de } \\
\text { la actividad }\end{array}$ & \\
\hline 4 & & & & & $\begin{array}{l}\text { Mejora } \\
\text { sistemas de } \\
\text { recogida en } \\
\text { estación y } \\
\text { entorno } \\
\end{array}$ & D & $\begin{array}{l}\text { Recogida de } \\
\text { residuos }\end{array}$ & $\mathrm{F}$ \\
\hline \multirow[t]{2}{*}{5} & \multirow{2}{*}{$\begin{array}{l}\text { Plan de actua- } \\
\text { ción y } \\
\text { seguimiento } \\
\text { del mismo }\end{array}$} & \multirow[t]{2}{*}{$\mathrm{D}$} & \multirow{2}{*}{$\begin{array}{l}\text { Recogida del } \\
\text { lijado de las } \\
\text { torres previo } \\
\text { ha ser pintadas }\end{array}$} & \multirow{2}{*}{ F } & \multirow{2}{*}{$\begin{array}{l}\text { Plan de } \\
\text { mantenimiento } \\
\text { sostenible }\end{array}$} & \multirow{2}{*}{ D } & Limpieza & D \\
\hline & & & & & & & $\begin{array}{l}\text { Mantenimiento } \\
\text { preventivo }\end{array}$ & $\mathrm{F}$ \\
\hline
\end{tabular}

\subsection{Interpretación de resultados: Matriz ICPA}




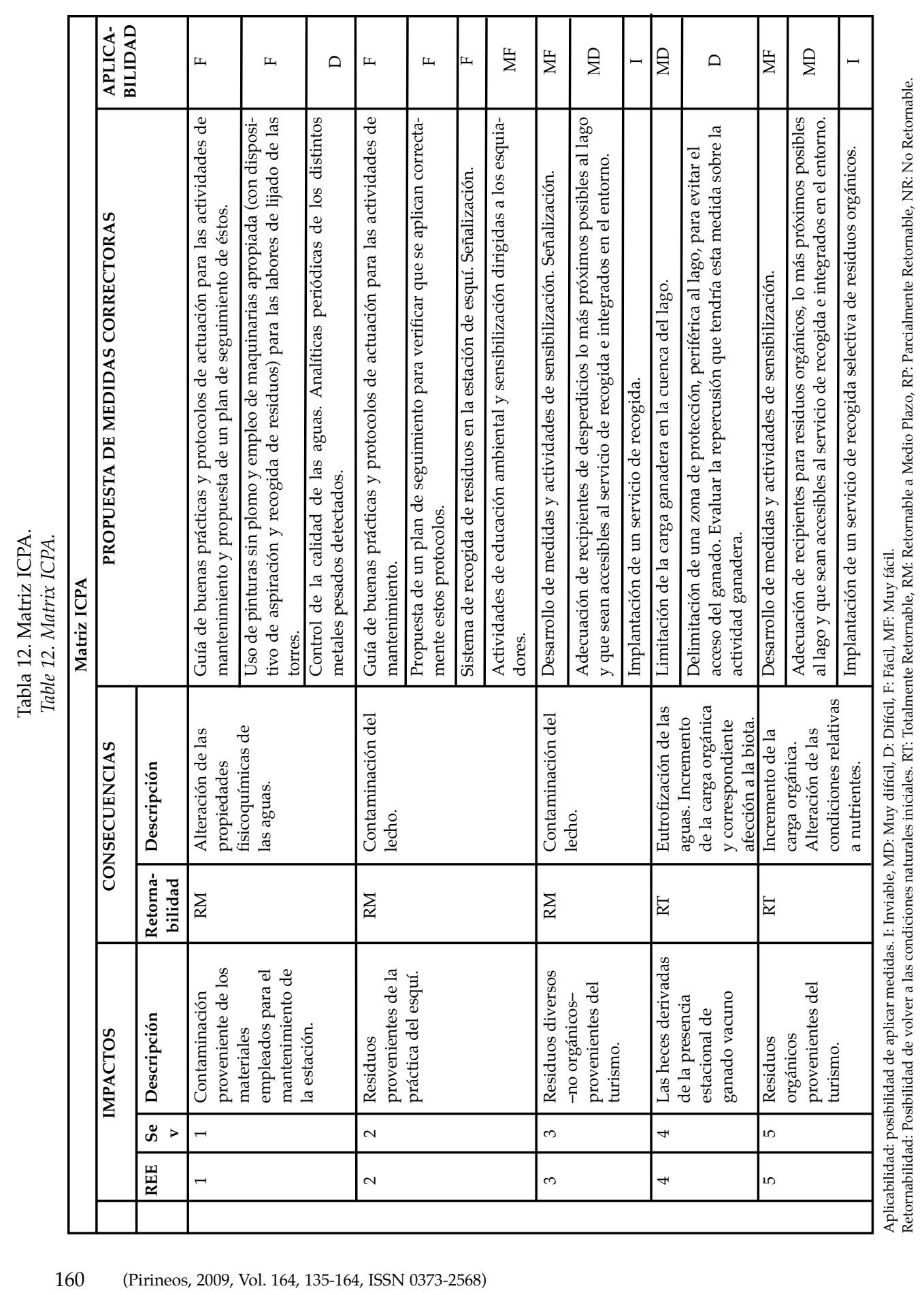




\section{Conclusiones}

El método propuesto es una herramienta útil y eficaz para la evaluación medioambiental de los lagos pirenaicos. Al basarse parcialmente en metodologías ya contrastadas en este campo, su uso no ha de presentar problemas de adaptación a los profesionales. Su correcta aplicación ha de quedar reflejada en un plan de medidas correctoras que permita mitigar las principales afecciones detectadas.

La calidad ecológica puede considerarse una buena aproximación al concepto de estado ecológico expuesto en la DMA, con la ventaja de su fácil operatividad y menor coste. Sin embargo, aplicar índices hidromorfológicos, junto con la valoración de otro tipo de indicadores bióticos (e.g, macrófitos, diatomeas, fitoplancton), sería necesario para obtener una visión más precisa del estado ecológico de estos ecosistemas.

De las distintas métricas contempladas en este estudio, las que se han considerado más adecuadas para su aplicación conjunta han sido InMacro, presencia de peces, Calidad Teórica y Calidad Adour-Garonne.

Si bien el IBMWP únicamente se ha empleado en puntos de aguas lóticas, podemos afirmar que este índice no es adecuado para la evaluación de estos lagos. De hecho, la obtención de un nuevo índice, similar al IBMWP y específico para los lagos pirenaicos, es prioritaria para poder determinar su estado ecológico.

Para determinar la calidad fisicoquímica se sugiere el empleo conjunto de los criterios expuestos por la Confederación Hidrográfica del Ebro, organismo de cuenca competente (i.e., CHE, 1990) y, considerando la proximidad a la cuenca de Adour-Garonne, los criterios que en ésta se proponen (ADOURGARONNE, 2006). Es destacable la diferencia existente entre umbrales de calidad propuestos por ambos organismos; la vulnerabilidad ecológica de estos lagos justifica la selección de los más restrictivos. Sería recomendable que existiesen criterios más homogéneos. Por ello, se sugiere la necesidad de establecer unos límites de calidad fisicoquímica consensuados, que permitan la comparación entre lagos pirenaicos que, aunque próximos, pertenezcan a cuencas hidrográficas distintas.

La calidad ecológica final de lago de Sabocos, aún despreciando los resultados del índice IBMWP, muestra unos resultados por debajo de lo esperado. La principal causa de tal determinación han sido las concentraciones de $\mathrm{Cr}$, $\mathrm{Pb}, \mathrm{Hg}$ y $\mathrm{Zn}$. En menor medida, también ha venido condicionada por los valores analizados de $\mathrm{DBO}_{5}, \mathrm{NH}_{4}^{+}, \mathrm{PO}_{4}^{-3}, \mathrm{O}_{2} \mathrm{y} \mathrm{DQO}$, todos ellos relacionados con la carga orgánica de las aguas.

Se ha de estudiar si las labores de mantenimiento de la estación de esquí se realizan bajo unas prácticas medioambientalmente adecuadas, gestionan- 
do correctamente los residuos peligrosos que se pudieran generar. No obstante, el origen de estos metales ha de ser estudiado en detalle aplicando modelos de transporte atmosférico. Del mismo modo, ampliar este planteamiento a distintos lagos de alta montaña es fundamental para caracterizar cualitativamente el estado ecológico de estos ecosistemas.

El principal agente responsable de la contaminación orgánica es el ganado. Así mismo, otras actividades (i.e., esquí, turismo) generadoras de un volumen considerable de residuos, también inciden sobre su estado de conservación.

La matriz ICPA del lago de Sabocos sintetiza las medias correctoras propuestas para mitigar las principales afecciones detectadas. Incluir dicha propuesta en la gestión de este entorno es fundamental para salvaguardar el estado ecológico del lago. Las actividades de educación y sensibilización ambiental, por la facilidad con pueden ser desarrolladas y llevas a la práctica, se convierten en una de las principales medidas que pueden contribuir a prevenir y corregir el deterioro de estos entornos.

\section{Agradecimientos}

Este estudio ha sido posible gracias a la ayuda que la Fundación Biodiversidad ha concedido al grupo de investigación GIMACES, de la Fundación San Valero. Así mismo, los autores desean mostrar su agradecimiento a la FARAS -de forma particular a José Manuel Cruz y Jorge Burgos-, a Universidad San Jorge, a la CHE, a Panticosa Resort, al Ayuntamiento de Panticosa, a Aramon Panticosa y al Grupo Especial de Actividades Subacuáticas de la Guardia Civil (GEAS). De un modo especial, se desea agradecer a los integrantes del panel de expertos - José Espona, Evelio Alvarez y José María Matesanz- y a Zoe Santolaria por su colaboración prestada en distintas fases del proyecto.

\section{Bibliografía}

AGENCE DE L'EAU ADOUR-GARONNE (2005). Bilan sur la qualité des eaux du Bassin Adour-Garonne en 2004, Anexo I, Les études de l'Agence de

l'Eau Adour-Garonne, 8 pp.

AGENCIA CATALANA DEL AGUA (2006). Protocolo de evaluación del estado ecológico de los lagos. $1^{\circ}$ Parte: manual de aplicación, Agencia Catalana del Agua, Cataluña, 21pp.

ALBA-TERCEDOR, J. y SANCHEZ-ORTEGA, A. (1988). Un método rápido y 
simple para evaluar la calidad biológica de las aguas corrientes basado en el de Helawell (1978), Limnética, 4: 51-56.

ARRUEBO, T., PARDO, A. y RODRÍGUEZ, C. (2006). Aproximación a un método sintético para la evaluación medioambiental de ecosistemas lacustres (Directiva 2000/60/CE). Actas VIII Congreso Nacional de Medio Ambiente, $16 \mathrm{pp}$.

CONESA, V. (1996). Instrumentos de la gestión ambiental en la empresa, Ediciones Mundi-Prensa, España, 541 pp.

CONFEDERACIÓN HIDROGRÂFICA DEL EBRO (1990). Definición de objetivos de calidad en función de los usos para las aguas superficiales de la cuenca del Ebro; Documento II: Establecimiento de la Calidad Teórica exigida en función de usos simultáneos, Ministerio de Obras Públicas y Urbanismo, Zaragoza.

CONFEDERACIÓN HIDROGRÁFICA DEL EBRO (2007). Cartografía - SIG. Accesible: oph.chebro.es/ ContenidoCartografico.htm, acceso: 13/02/ 2007.

DIRECTIVA MARCO DEL AGUA. Directiva 2000/60/CE de la Unión Europea y del Consejo, de 23 de Octubre de 2000, por la que se establece un marco comunitario de actuación en el ámbito de la política de Aguas, Diario Oficial L 327 de 22.12.2000, 72 pp.

GARRIDO GONZÁLEZ, J., MEMBIELA IGLESIAS, P. y VIDAL LÓPEZ, M. (1998). Calidad biológica de las aguas del río Barbaña, Tecnología del Agua, 175, pp: 50-54.

GÓMEZ OREA, D. (2003). Evaluación de Impacto Ambiental, Ediciones MundiPrensa, España, 749 pp.

INSTITUTO ARAGONÉS DE ESTADÍSTICA (2007). Accesible: http:// bonansa.aragon.es: 81 /estadis/base_ter/bas_consulta.jsp?tema= $1 \&$ terri $=4 \&$ codigo $=22170$, acceso: $25 / 04 / 2007$.

LANAJA, F.J., ARRUEBO, T., PARDO, A. y RODRÍGUEZ, C. (2005). Evaluación de la calidad ecológica de un lago glaciar pirenaico (Ibón) afectado por la acción antrópica, Tecnología del Agua, 266, pp: 66-72.

LARRY N. C. (1999). Manual de Evaluación de Impacto Ambiental. Técnicas para la elaboración de Estudios de Impacto, 841 pp.

LECLERCQ. L. (2001). Interet et limites des méthodes d'estimation de la qualité de l'eau. Statio scientifique des Haustes-Fagnes, rue de Botrange, 137, Robertville, Bélgica.

LEOPOLD, L.B., CLARKE, F.E., HANSHAW, B. B., y BALSLEY, J.R. (1971). A procedure for Evaluation Environmental Impacts, U. S. Geological Survey, Circular 645, United State Environmental Protection Agency, Washington, $13 \mathrm{pp}$.

MAST, M.A., CAMPBELL, D.H., INGERSOLL, G.P., FOREMAN, W.T., y KRABBENHOFT, D.P. (2003), Atmospheric Deposition of Nutrients, 
Pesticides, and Mercury in RockyMountain National Park, Colorado, U.S. Department of the Interior and U.S. Geological Survey, Water-Resources Investigations Report 03-4241, Virginia, $15 \mathrm{pp}$.

MUÑOZ, I.; PICÓN, A; SABATER, S. y ARMENGOL J. (1998). La calidad del Agua del río Ter a partir del uso de índices biológicos, Tecnología del Agua, 175, pp: 60-67.

SISTEMA DE INFORMACIÓN TERRITORIAL DE ARAGÓN (2007). Accesible: sitar.aragon.es, acceso: 17/05/2007.

PRAT, N. y MUNNÉ, A. (1999). Delimitación de regiones ecológicas de la cuenca del Ebro, Departamento de Ecología de la Universidad de Barcelona. Accesible: www.oph. chebro.es/DOCUMENTACION/EstudiosEcologicos/1998-Ph-08-I/index.htm, acceso: 20/12/2004

RIVAS MARTÍNEZ, S., BÁSCONES, J. DÍAZ, T., FERNÁNDEZ, F., y LOIDI, J. (1991). Vegetación del Pirineo Occidental y Navarra, Itinera Geobotánica, 5: $5-456$.

RODRÍGUEZ, C., ARRUEBO, T. y PARDO, A. (2009). Modelo de gestión para espacios naturales de alto interés ecológico: lagos de alta montaña (ibones). Publicaciones del Consejo de Protección de la Naturaleza de Aragón, Zaragoza, en prensa.

VAN DROOGE, B., GRIMALT, J., TORRES, C., y CUEVAS, E. (2002). Semivolatile Organochlorine Compounds in the Free Troposphere of the Northeastern Atlantic, Environmental Science and Technology, 36, 6, pp: 11551161.

VAL HERNÁNDEZ, M. (2002). Estudio de calidad ecologica del río Aragón desde su nacimiento en el valle de Astún hasta Jaca (Huesca), Consejo de Protección de la Naturaleza de Aragón, Zaragoza, 199 pp. 